\title{
Universal risk assessment upon hospital admission for screening of carriage with multidrug-resistant microorganisms (MDRO) in a Dutch tertiary care centre $(2016-2019)$
}

Denise van Hout ( $\boldsymbol{D}$ d.vanhout-3@umcutrecht.nl ) Universitair Medisch Centrum Utrecht https://orcid.org/0000-0003-0579-8149

Patricia Bruijning-Verhagen

Universitair Medisch Centrum Utrecht

Hetty Blok

Universitair Medisch Centrum Utrecht

Annet Troelstra

Universitair Medisch Centrum Utrecht

Marc Bonten

Universitair Medisch Centrum Utrecht

\section{Research}

Keywords: multi-drug resistant organisms (MDRO), risk assessment, hospitalization, The Netherlands,

Posted Date: March 11th, 2020

DOI: https://doi.org/10.21203/rs.3.rs-16670/v1

License: (c) (i) This work is licensed under a Creative Commons Attribution 4.0 International License.

Read Full License 


\section{Abstract}

\section{Background}

In Dutch hospitals a 6-point questionnaire is mandatory for risk-assessment to identify carriers of multidrug-resistant organisms (MDRO) at the time of hospitalization. Presence of one or more risk factors is followed by microbiological culturing and pre-emptive isolation. We evaluated the test characteristics of this screening tool in identifying new MDRO carriers.

\section{Methods}

A cross-sectional study using routinely collected healthcare data was performed in a Dutch tertiary hospital between 1 January 2015 and 1 August 2019 including all admissions with an MDRO risk assessment performed on the day of admission. MDRO risk-assessment included: (1) known MDRO carriage, (2) previous hospitalization in another Dutch hospital during a known outbreak, (3) previous hospitalization in a foreign hospital, (4) living in an asylum centre, (5) professional exposure to livestock farming and (6) household membership of a methicillin-resistant Staphylococcus aureus (MRSA) carrier. Sensitivity of the risk assessment was estimated by comparing observed prevalence of newly detected MDRO carriage to expected prevalence of carriage in the Dutch population upon hospital admission.

Results

144,051 hospital admissions of 84,485 unique patients were included. In total, 4,480 (3.1\%) admissions had a positive MDRO risk-assessment (i.e. $\geq 1$ risk factors present). In 1,516 (34\%) admissions microbiological screening was performed, of which 341 (23\%) yielded MDRO. 81 patients were categorized as new MDRO carriers, as identified through MDRO risk-assessment, reflecting $0.06 \%(95 \% \mathrm{Cl}$ : $0.04 \%-0.07 \%)$ of all admissions and $1.8 \%(95 \% \mathrm{Cl}: 1.4 \%-2.2 \%)$ of those with positive risk assessment. MDRO included ESBL-producing and/or multidrug-resistant Enterobacterales $(n=52,64 \%)$, MRSA ( $n=26$, $32 \%)$, carbapenem-resistant Enterobacterales (CRE) $(n=2,3 \%)$ and VRE $(n=1,1 \%)$. The numbers of "MDRO risk-assessments needed to perform" and individual "MDRO risk-assessment questions needed to ask" to detect one new MDRO carrier upon admission were 1,778 and 10,420, respectively. Estimated sensitivities of the risk-assessment for detecting MDRO carriage were $<1 \%$, for ESBL-E and VRE, $<2 \%$ for CRE and $18 \%$ for MRSA.

\section{Conclusions}

The number of risk-assessments needed to perform to detect one new MDRO carrier upon hospital admission was high, and the vast majority of carriers most likely remained undetected. The current MDRO risk assessment upon admission strategy needs thorough reconsideration.

\section{Background}


Dissemination of multidrug-resistant microorganisms (MDRO) in healthcare settings may lead to more infections caused by MDRO, which may reduce effectiveness of empirical antibiotic therapy (1-4). The hospital setting facilitates patient-to-patient transmission of MDRO because of the high antibiotic selective pressure, frequent contact between healthcare workers and patients and vulnerability of patients to acquire carriage with MDRO. Optimizing control strategies is, therefore, important to prevent dissemination and associated risks of infections caused by MDRO. Hospital-based surveillance is recommended for timely detection of MDRO carriage and installation of transmission-based contact precautions. In the Netherlands, hospitals have adopted a risk-based screening for asymptomatic MDRO carriage upon admission. This originated in the mid-1980s to control the emergence of methicillinresistant S. aureus (MRSA), as one of the elements of the Dutch 'search and destroy' strategy (5-10). Over the years, this risk-based screening was extended to also control other MDRO, such as multidrugresistant Gram-negative bacteria (MDR-GNB) $(11,12)$. MDRO risk assessment is, for each patient, based on a 6-point questionnaire that needs to be checked upon admission. These questions include risk factors for carriage of MRSA, MDR-GNB, and vancomycin-resistant enterococci (VRE). In patients at risk of MDRO carriage, according to this screening, pre-emptive contact precautions should be installed and screening cultures should be obtained. Adherence to this strategy is monitored by the Dutch Healthcare Inspectorate. Yet, this approach requires time for questioning patients, pre-emptive isolation measures that may affect care of other patients and resources for microbiological testing. The benefits of the strategy have not yet been quantified.

The aim of the current study was to evaluate the current risk assessment for screening of MDRO carriage upon hospital admission in a Dutch tertiary care hospital. We, therefore, determined the number of newly identified MDRO carriers and the number of questions needed to ask to identify one new MDRO carrier. We also compared the detected prevalence of MDRO carriage with the expected prevalence of MDRO carriage in the Dutch population upon hospital admission.

\section{Methods}

\section{Study design}

This observational study was performed in the University Medical Center Utrecht (UMCU) in the Netherlands. The UMCU is a tertiary care medical centre with 1,042 beds for adults and children, all medical specialties represented and around 180,000 inpatient days per year. A cross-sectional study using routinely collected healthcare data was performed of all hospital admissions between 1 January 2015 and 1 August 2019. For this study we extracted data from all hospital admissions with completion of the MDRO risk assessment in the electronic medical record (EMR) on the same day as hospitalization. A hospital admission was defined as any admission to any ward, including admissions for single-day treatments, and for all ages. Characteristics available per admission were age, sex and length of stay (LOS). Results of this study were reported following the Strengthening the Reporting of Observational studies in Epidemiology (STROBE) criteria (13). 


\section{MDRO risk assessment}

The screening strategy consisted of two consecutive steps. Step one was an individual 6-item risk assessment for MDRO carriage. The six questions referred to (1) known MDRO carriage, (2) previous hospitalization in another Dutch hospital during the past 2 months with an ongoing outbreak during hospitalization, (3) previous hospitalization in a foreign hospital in the past 2 months, (4) living in an asylum shelter, (5) professional exposure to livestock farming (i.e. living pigs, veal calves or broilers), and (6) living with a known MRSA carrier (the entire questionnaire is provided in Additional File 1). The MDROassessment was obligatory and embedded in the EMR, to be completed within 24 hours for each patient admitted, visiting the emergency department or out-patient clinic for pre-operative screening. Answers of the assessment remained valid for 62 days after completion and answers were automatically completed if a new assessment was started within this time-window. In case of more than one MDRO-assessment obtained on the day of admission, only the first one was used for the current study. A positive MDRO risk assessment was defined as at least one question answered with 'yes'. A positive assessment automatically generated an isolation label in the EMR with a responsive order for pre-emptive contact precautions for that patient. The second step entailed obtaining screening cultures from these patients,

unless someone was a known carrier and/or there were culture results with MDRO that had been obtained in the past 2 months. Screening cultures were routinely assessed for growth of MDR-GNB and MRSA; other MDRO were assessed upon indication (e.g. previous carriage, outbreak in previous hospital). If screening cultures yielded MDRO, contact precautions were continued and if not, the EMR isolation label was removed and contact precautions were discontinued. All steps were coordinated semi-automatically by the Infection Prevention (IP) specialists, who manually reviewed positive MDRO-assessments within 24 hours and who modified infection control measures, where needed. IP specialist were also automatically notified in case of any (screening or clinical) culture yielding MDRO and manually assigned isolation labels in the EMR if contact precautions were needed.

\section{Microbiology}

We defined screening cultures as nasal, throat, rectal or perineal swabs obtained at the day of admission or the day thereafter in patients with a positive MDRO-assessment (See Table S2, Additional File 2). MDRO included MRSA, VRE, extended-spectrum beta-lactamase (ESBL)-producing and/or multidrugresistant Enterobacterales (ESBL/MDR-E), carbapenem-resistant Enterobacterales (CRE), multidrugresistant Acinetobacter spp (MDR-A), carbapenem-resistant Acinetobacter spp (CRA), multi-drug resistant P. aeruginosa, cotrimoxazol-resistant S. maltophilia and penicillin-resistant S. pneumoniae (PSP) (See Table S3, Additional File 3 for definitions). The categories ESBL/MDR-E and CRE were mutually exclusive (i.e. strains categorized as ESBL/MDR-E were not carbapenem-resistant, because these were categorized separately). Definitions of MDRO were based on the Dutch Working Party Infection Prevention (WIP) guidelines and were adapted to local definitions of the UMCU if applicable (12).

\section{Statistical analyses}


We determined 'the number of MDRO risk assessments to perform' and 'the number of MDROassessment questions needed to ask' to detect one new MDRO carrier upon hospital admission by dividing the total number of admissions and the corresponding MDRO-assessment questions by the total number of newly identified MDRO carriers, respectively. The positive predictive value (PPV) was determined for each of the individual questions of the MDRO-assessment. The PPV was calculated as the number of admissions in which the question was answered positively and screening identified new MDRO carriage, divided by the total number of times the question was answered positively. Naturally, patients admitted might already have an isolation label in the EMR (usually based upon prior culture results), yet, in routine care, such patients are also part of the risk assessment. We, therefore, determined in admissions with a positive MDRO-assessment and with MDRO in screening cultures the presence of prior isolation labels in the EMR. The observed prevalence of detected MDRO carriage through risk assessment was compared to expected MDRO carriage of the Dutch population, based on recent studies (if available; of the last 10 years), to estimate the sensitivity of the risk assessment and the proportion of MDRO carriers that still remained undetected upon admission.

False-positive risk assessment leads to unnecessary (pre-emptive) isolation days until screening cultures turn out to be negative for MDRO. In the absence of our risk assessment strategy, true positives would remain undetected until clinical cultures yield MDRO or until patient discharge. We, therefore, determined the average length of stay until the first clinical culture yielding MDRO for admissions with newly identified MDRO carriage identified through risk assessment. In absence of MDRO in clinical cultures the total duration of hospital stay was used. These days were used as a proxy of the maximum duration of pre-emptive contact precautions gained by the screening strategy. The total number of unjustified isolation days was calculated as the total number of isolation days until negative screening results were available, for which we assumed 0.5 days for MRSA (based upon PCR testing of nasal swabs) and 1.5 days for other MDRO (based upon conventional cultures).

Data were reported with means \pm standard deviation (SD), medians with first and third quartile $\left(\mathrm{Q}_{1}-\mathrm{Q}_{3}\right)$ or percentages, where appropriate. $95 \%$ confidence intervals $(\mathrm{Cl})$ of proportions were calculated using the Exact method (14). All statistical analyses were performed with Statistical Package for Social Sciences V.25.0.2 (SPSS, Chicago, Illinois, USA) and R Version 3.4.1.

\section{Results}

\section{Patient population}

In all, 171,974 MDRO assessments of non-cancelled admissions were obtained. As two or more assessments were obtained in 27,923 (16.2\%) admissions, exclusion of duplicate assessments led to 144,051 hospital admissions of 84,485 unique patients for analysis (Fig. 1). MDRO risk assessment was performed on the day of admission in $90.3 \%$ of hospital admissions. The median age of admissions was 49 years $\left(Q_{1}-Q_{3} 19-67\right)$ and $48 \%$ were female. Median length of stay (LOS) was 1 day $\left(Q_{1}-Q_{3} 0-4\right)$ and $64.6 \%$ of all admissions included an overnight stay. 


\section{Identification of new MDRO carriers}

In total, 4,480 (3.1\%) admissions had a positive MDRO-assessment and pre-emptive contact precautions installed, which was mainly based on the presence of known carriage with MDRO ( $n=3,206,71.6 \%)$ (Table 1). In 1,516 (33.8\%) of these admissions screening cultures were obtained, of which 341 (22.5\%) yielded MDRO (Fig. 1). Predominant reasons for not obtaining screening cultures were known MDRO carriage status $(77.8 \%)$ or re-categorization to low risk by IP specialists $(13.7 \%)$. Of the remaining 253 (8.5\%) episodes, discharge was on the same day as admission in $109(3.7 \%)$ and reasons for not obtaining screening cultures were unknown in 144 (4.9\%) admissions.

In $260(76.2 \%)$ admissions with MDRO growing in screening cultures an isolation label was already present in the EMR at the time of hospitalization (of which 29 (11.1\%) due to a previous risk assessment). In all, 81 admissions (of 81 unique patients) were categorized as newly identified MDRO carriers due to the MDRO risk assessment screening strategy (Fig. 1). This reflects $0.06 \%(95 \% \mathrm{Cl}: 0.04 \%-0.07 \%$ ) of all admissions and $1.8 \%$ (95\% Cl: $1.4 \%-2.2 \%$ ) of all admissions with a positive risk assessment. Of these, 52 (64.2\%) carried MDR-E/ESBL-E and $26(32.1 \%)$ carried MRSA. MDR-E isolates $(n=33)$ were resistant to fluoroquinolones and aminoglycosides, but susceptible to carbapenems. The MDRO risk assessment strategy identified CRE carriage (rectal carriage with OXA-48-like Enterobacter cloacae and OXA-48 Klebsiella pneumoniae, respectively) in two patients with recent hospitalization abroad, and one VRE carrier with known carriage due to screening in another hospital. The number of newly identified MDRO carriers through risk-based screening was stable over time (See Table S4, Additional File 4) and the identified MDRO per risk factor is provided in Table S5 (Additional File 5).

\section{MDRO risk assessment}

Positive predictive values of the individual questions for identifying new MDRO carriage ranged from $1.0 \%$ (95\% Cl: $0.7 \%-1.3 \%$ ) for "Are you a known carrier of an MDRO?" to $7.0 \%$ (95\% Cl: $4.3 \%-11.1 \%$ ) for "Did you live in an asylum shelter during the past 2 months?" (Table 1). Yet, the number needed to ask of the individual questions to detect one new MDRO carrier ranged from 4,647 for "Are you a known carrier of an MDRO?" to 71,563 for "Are you a household member of an MRSA carrier?". The numbers of 'MDRO risk assessments needed to perform' and individual 'MDRO-questions needed to ask' to detect one new MDRO carrier upon hospital admission were $1,778(144,041 / 81)$ and 10,420 (844,031/81), respectively.

When comparing the observed prevalence of newly identified carriers based on the screening strategy to the perceived prevalence of MDRO carriage upon hospital admission based on recent epidemiological studies in the Netherlands, estimated sensitivities of the risk assessment for detecting MDRO carriage were $<1 \%$ for ESBL-E and VRE, $<2 \%$ for CRE and $18.2 \%$ for MRSA carriage (Table 2 ). 
Table 1

Admissions with positive MDRO risk assessment, positive MDRO screening cultures and positive predictive value for new identified MDRO carriage per question.

\begin{tabular}{|c|c|c|c|c|c|}
\hline & $\begin{array}{l}\text { Answered } \\
\text { positively, } \\
\mathrm{n}\left(\%^{\mathrm{a}}\right)\end{array}$ & $\begin{array}{l}\text { Screening } \\
\text { cultures } \\
\text { obtained, } n \\
\text { (\%) }\end{array}$ & $\begin{array}{l}\text { New } \\
\text { identified } \\
\text { MDRO } \\
\text { carriage, } n \\
\left(\%^{b}\right)\end{array}$ & $\begin{array}{l}\text { PPV } \\
(\%) \\
(95 \% \\
\text { Cl) }\end{array}$ & $N_{N A}{ }^{c}$ \\
\hline $\begin{array}{l}\text { 1. Are you a known carrier of an MDRO } \\
\text { (e.g. MRSA, VRE, MDR-GNR)? }\end{array}$ & $\begin{array}{l}3,206 \\
(2.8)\end{array}$ & 901 (28.1) & $31(3.4)$ & $\begin{array}{l}1.0 \\
(0.7- \\
1.3)\end{array}$ & 4,647 \\
\hline $\begin{array}{l}\text { 2. During the past } 2 \text { months, were you } \\
\text { hospitalized in another Dutch hospital } \\
\text { during a known MDRO outbreak? }\end{array}$ & $200(0.1)$ & $46(23.0)$ & $3(6.5)$ & $\begin{array}{l}1.5 \\
(0.5- \\
4.4)\end{array}$ & 47,798 \\
\hline $\begin{array}{l}\text { 3. During the past } 2 \text { months, were you } \\
\text { hospitalized in a foreign hospital? }\end{array}$ & $673(0.5)$ & $372(55.3)$ & $34(9.1)$ & $\begin{array}{l}5.0 \\
(3.9- \\
6.5)\end{array}$ & 4,228 \\
\hline $\begin{array}{l}\text { 4. In the past } 2 \text { months, did you live in } \\
\text { an asylum shelter? }\end{array}$ & $187(0.2)$ & $106(56.7)$ & $13(12.3)$ & $\begin{array}{l}7.0 \\
(4.3- \\
11.1)\end{array}$ & 8,875 \\
\hline $\begin{array}{l}\text { 5. Do you work with living pigs, veal } \\
\text { calves or broilers? }\end{array}$ & $340(0.2)$ & $141(41.5)$ & $8(5.7)$ & $\begin{array}{l}2.4 \\
(1.2- \\
4.5)\end{array}$ & 17,919 \\
\hline $\begin{array}{l}\text { 6. Are you a household member of an } \\
\text { MRSA carrier? } \\
\text { One or more of six questions answered } \\
\text { positively }\end{array}$ & $\begin{array}{l}116(0.1) \\
4,480 \\
(3.1)\end{array}$ & $\begin{array}{l}54(46.6) \\
1,516 \\
(33.8)\end{array}$ & $\begin{array}{l}2(3.7) \\
81(5.3)\end{array}$ & $\begin{array}{l}1.7 \\
(0.4- \\
6.5) \\
1.8 \\
(1.5- \\
2.2)\end{array}$ & $\begin{array}{l}71,563 \\
1,778\end{array}$ \\
\hline \multicolumn{6}{|c|}{$\begin{array}{l}\mathrm{Cl} \text {, confidence interval; MDRO, multi-drug resistant organism; MDR-GNR, multi-drug resistant Gram- } \\
\text { negative rod; MRSA, methicillin-resistant S. aureus; NNA, number needed to ask; PPV, positive } \\
\text { predictive value; VRE, vancomycin-resistant Enterococcus. } \\
\text { a. Proportion of the total number of times the particular question was asked. Missings were excluded } \\
\text { from the denominator. } \\
\text { b. Individual column counts count up to > } 81 \text { because risk assessment could have a positive reply to } \\
\text { multiple questions. } \\
\text { c. "Number of questions needed to ask"; calculated as the total number of times the question was } \\
\text { asked divided by the number of newly identified MDRO carriers with a positive reply to this question. }\end{array}$} \\
\hline
\end{tabular}

MDRO in clinical cultures during hospital stay

In 1,279 (0.9\%) hospital admissions clinical cultures yielded MDRO during hospital stay, and 765 (59.8\%) of these admissions had negative risk assessments at the time of admission (See Table S6 and S7, Additional File 6). In 12 (14.8\%) of the 81 admissions with newly identified MDRO carriage, the same type of MDRO was also identified in clinical cultures during hospital stay. For these 12 patients, the median 
LOS until MDRO detection in clinical cultures was 4 days $\left(Q_{1}-Q_{3} 2-6\right)$, and the total number of days was 53. Most clinical cultures were from urine $(n=5,29.4 \%)$ (See Table S8, Additional File 6$)$. The total LOS of the 69 MDRO carriers that would not have been detected without risk-based screening was 513, making 566 days of unprotected ward stay that was prevented by the screening strategy. The total number of unjustified isolation days due false-positive risk assessment was 1,436 days.

Table 2

Sensitivity of risk assessment strategy for detecting MDRO carriage at the time of hospital admission.

\begin{tabular}{|c|c|c|c|}
\hline & $\begin{array}{l}\text { Prevalence of newly identified } \\
\text { carriage upon admission by } \\
\text { risk-based screening ( } 95 \% \mathrm{Cl}) \\
\text { - current study }(\%)\end{array}$ & $\begin{array}{l}\text { Reported prevalence of } \\
\text { carriage upon admission } \\
\text { in other Dutch studies } \\
\text { (\%) }\end{array}$ & $\begin{array}{l}\text { Estimated } \\
\text { proportion } \\
\text { detected by risk- } \\
\text { based screening } \\
\text { (\%) }\end{array}$ \\
\hline $\begin{array}{l}\text { ESBL-positive } \\
\text { Enterobacterales }\end{array}$ & $0.03(0.02-0.04)$ & 6.4 to $7.0(16)$ & 0.4 to 0.5 \\
\hline MRSA & $0.02(0.01-0.03)$ & 0.11 (17) to $0.13(18)$ & 15.4 to 18.2 \\
\hline $\begin{array}{l}\text { Carbapenem-R } \\
\text { Enterobacterales }\end{array}$ & $0.001(0.0002-0.005)$ & $\begin{array}{l}<0.06 \text { (19) to } 0.25(20- \\
22)\end{array}$ & 0.4 to 1.7 \\
\hline VRE & $0.0007(0.00002-0.004)$ & $1.3(22)$ to $1.5^{a}(23,24)$ & 0.05 \\
\hline \multicolumn{4}{|c|}{$\begin{array}{l}\text { ESBL, extended-spectrum beta-lactamase; MDRO, multidrug-resistant organism; MRSA, methicillin- } \\
\text { resistant S. aureus; R, resistant; VRE, vancomycin-resistant Enterococcus } \\
\text { a. NB. Estimates for VRE carriage derive from point-prevalence surveys in patients during admission } \\
\text { and a population-based study on community intestinal carriage. }\end{array}$} \\
\hline
\end{tabular}

\section{Discussion}

In this analysis of 144,051 hospital admissions a strategy of risk-based screening for MDRO carriage upon hospital admission identified previously unknown MDRO carriage in $0.06 \%$ (95\% Cl: $0.04 \%-0.07 \%$ ) of all admissions and in $1.8 \%$ (95\% $\mathrm{Cl}: 1.4 \%-2.2 \%)$ of all patients considered to be at high risk of MDRO carriage. The numbers of 'MDRO risk assessments needed to perform' and individual 'MDRO risk assessment questions needed to ask' to detect one new MDRO carrier upon hospital admission were 1,778 and 10,420, respectively. Still, the vast majority of MDRO carriers most likely remained undetected.

The calculated numbers needed to ask are actually even underestimated as $16 \%$ of admissions had more than one MDRO risk assessment completed on the same day, and these copy-assessments were excluded from our analysis. If included, the numbers of "MDRO risk assessments actually performed" and "MDRO risk assessment questions actually asked" to detect one new MDRO carrier upon admission would have been $2,123(171,974 / 81)$ and $12,440(1,007,640 / 81)$, respectively. If we, conservatively, estimate one minute of labour time per MDRO risk assessment and one minute for administration, at least 160 36-hour working weeks were spent on performing assessments during these four and a half 
years. This reflects at least two working weeks spent per newly identifed MDRO carrier (160 weeks divided by 81 new carriers).

Newly identified carriers were most often colonized with ESBL-producing and/or Enterobacterales strains resistant to both an aminoglycoside and ciprofloxacin (70\%), MDRO of which the value of screening upon admission for the prevention of transmission and hospital-acquired infections is not well-established (25-27). In our study, the prevalence of newly detected ESBL carriage upon admission was $0.03 \%$ (95\% $\mathrm{Cl}$ : 0.02-0.04), which is considerably lower than the prevalence of faecal ESBL carriage in the Dutch community; which was $5 \%$ in randomly selected subjects (19) and $6.4-7.0 \%$ upon admission to our hospital (16). As a result, in our hospital, the proportion of ESBL carriers that still remained undetected upon admission despite risk-based screening was probably more than $99 \%$. For CRE and VRE the proportion of undetected carriers was equally high, being $>98 \%$ and $>99 \%$, respectively.

The second most common MDRO in new carriers was MRSA (26\%), which was identified in $0.02 \%$ (95\% Cl: $0.01 \%-0.03 \%)$ of all admissions. Screening and pre-emptive isolation of high-risk patients for MRSA has been an important part of the Dutch 'search and destroy' policy for the prevention of MRSA transmission (6-8,17,28-31). In our study, positive predictive values to detect - among others - MRSA carriage ranged from $2.4 \%$ (95\% Cl: $1.2 \%-4.5 \%$ ) (working with living pigs, veal calves or broilers) to $5.0 \%$ (95\% Cl: 3.9\%-6.5\%) (previous hospitalization in a foreign hospital). Still, presence of these risk factors was rare and even lowest for the question about being a household member of an MRSA carrier $(0.1 \%)$, which needed to be asked 71,563 times in order to identify one new MRSA carrier upon hospital admission. In a recent analysis of routine universal pre-operative screening for nasal S. aureus carriage during a 7-year period in another Dutch hospital the prevalence of MRSA carriage was $0.13 \%$, comparable to the reported prevalence of $0.11 \%$ upon admission in a study performed eight years earlier $(17,18)$. Assuming a similar prevalence in patients admitted to our hospital would imply that the current screening strategy identified only $15 \%$ of all MRSA carriers upon admission, suggesting that $85 \%$ still remained undetected. This is in line with other studies that report that currently most MRSA carriers do not have the classical risk factors (i.e. as inquired with our risk assessment) for MRSA carriage (7,32-34).

The assessment question on known MDRO carriage had the highest yield, as it was answered positively in $2.8 \%$. Indeed, $76 \%(n=260)$ of all patients with an MDRO positive screening culture were already labelled in our EMR as a known MDRO carrier, of which $11 \%(n=29)$ had this label due to previous riskbased screening. This implies that if the risk assessment would have been replaced by the use of existing MDRO labelling in the EMR $68 \%(231 / 341)$ of MDRO carriers - that were now identified by risk-based screening - would still be captured.

Typically, the unexpected identification of an MDRO carrier during admission (i.e. through a positive clinical culture) is associated with extra workload, for screening of exposed roommates or healthcare workers of the index patient. This is not needed if the carrier was already identified upon admission (and thus contact precautions had already been installed). In our study only $15 \%(n=12)$ of detected carriers had a clinical culture positive for MDRO during admission, for which contact tracing would have been 
implemented if screening upon admission had not been applied. We estimated that abandoning riskassessment based screening would have led to 566 patient days without protective measures for MDRO carriers in the 4.5 years of the observation period. As the vast majority of MDRO carriers remained undetected, these 566 days add little to the total number of patient days without protective measures for - unknown - MDRO carriers. The number of prevented episodes of cross-transmission due to the identification of new MDRO carriers upon admission is difficult to determine. Yet, the total number of hospital-acquired bacteraemia episodes caused by MDRO in our hospital during the 54 months of the study period was $44 ; 37$ were caused by ESBL/MDR-Enterobacterales, four by multidrug-resistant $P$. aeruginosa, two by MRSA and one by VRE. Of these 44 patients eleven were known MDRO carriers at the time of admission and two were newly identified as MDRO carrier through risk assessment and screening. The remaining 31 patients (including those with hospital-acquired bacteraemia caused by MRSA and VRE) had no risk factors for MDRO carriage upon admission.

A strength of the current analysis was the combination of routine care data and medical microbiology information of $90 \%$ of all admissions during the predefined study period. There are also important limitations of this study that should be acknowledged. Firstly, retrograde manual changes to the MDRO risk assessment during hospital admission could not be retrieved. It is, therefore, not excluded that the MDRO risk assessment (e.g. the first question) was manually changed to 'positive' in case of MDRO positive cultures during admission. If so, the value of MDRO risk assessment would have been overestimated. Secondly, this was a real-life evaluation of clinical practice, without confirmation whether the individual questions of the MDRO risk assessment were answered correctly. Thirdly, this was a singlecentre analysis in an academic medical centre in the Netherlands, with a well-developed system of identification, labelling and isolation of MDRO carriers as well as good adherence to standard precautions in routine care. Extrapolation of findings to other settings or countries should always occur in light of local epidemiology and established routine infection and prevention practices within a hospital.

The current low levels of AMR in hospitals in the Netherlands are partly explained by a restrictive use of antibiotics combined with the well-established 'search and destroy' policy over the last decades. Still, critical appraisal as well as continuous improvement is a fundamental part of infection prevention and control, considering that local epidemiology and target populations may change over time. Results of this study imply that the majority of MDRO carriers in the community remains undetected upon admission despite current risk-based screening. Combined with the low prevalence of risk factors and the types of MDRO that are most often identified, the question arises whether the number of newly identified MDRO carriers truly justifies the invested workload across all hospital wards in risk assessment upon admission. We propose a system in which risk-based screening is abandoned and instead, contact precautions are installed upon hospitalization of patients that are known (previous) carriers of MDRO. This captures the majority of MDRO carriers that would elsewise be identified through risk assessment.

\section{Conclusions}


In conclusion, in an academic Dutch hospital with a well-established MDRO surveillance system, individual risk assessment and screening for MDRO carriage upon hospital admission resulted in a low yield of new identified MDRO carriers in comparison to overall invested workload, while the majority of carriers remained undetected. Our findings justify a thorough reconsideration of the current individual risk assessment for MDRO carriage upon admission.

\section{Declarations}

\section{Ethics approval and consent to participate}

This study was performed in line with the Declaration of Helsinki, as revised in 2013 (15). Because this study does not fall under the scope of the Medical Research Involving Human Subjects Act (in Dutch: 'WMO'), the Medical Research Ethics Committee of the UMCU waived the need for official approval by the UMCU Ethics Committee (IRB correspondence number 18-574C) and individual informed consent was not obtained. All data were analyzed and stored pseudonymized.

\section{Consent for publication}

In line with ARIC editorial policies consent for publication of individual person's data (i.e. names) was given by all persons who this applied to for the current article (i.e. co-authors and persons mentioned in the Acknowledgements section).

\section{Availability of data and materials}

The datasets used and/or analysed during the current study are available from the corresponding author on reasonable request.

\section{Competing interests}

The authors declare that they have no competing interests.

\section{Funding}

This study was internally funded by the University Medical Center Utrecht (UMCU) and the authors received no specific funding for this work.

\section{Authors' contributions}

$\mathrm{DH}, \mathrm{PB}, \mathrm{HB}, \mathrm{AT}$ and $\mathrm{MB}$ contributed to study design and data collection. DH performed the statistical analyses and drafted the first version of the manuscript. All authors contributed to data interpretation and manuscript editing. All authors read and approved the final manuscript.

\section{Acknowledgements}


We sincerely thank Roel Elbers for his important part in the data-extraction and data-management part of the current study. We are grateful for the assistance of Maaike van Mourik in the transfer of the risk assessment data to the UMCU Research Data Platform that was needed to start the study.

\section{Authors' information}

Julius Center for Health Sciences and Primary Care, University Medical Center Utrecht, Utrecht University, Utrecht, The Netherlands

Denise van Hout, Patricia Bruijning-Verhagen, Marc Bonten

Center for Infectious Disease Control, National Institute for Public Health and the Environment, Bilthoven, The Netherlands

Patricia Bruijning-Verhagen, Marc Bonten

Department of Medical Microbiology, University Medical Center Utrecht, Utrecht, Utrecht University, The Netherlands

Hetty Blok, Annet Troelstra, Marc Bonten

\section{References}

1. Mehl A, Asvold BO, Kummel A, Lydersen S, Paulsen J, Haugan I, et al. Trends in antimicrobial resistance and empiric antibiotic therapy of bloodstream infections at a general hospital in MidNorway: a prospective observational study. BMC Infect Dis. 2017;17(1):116.

2. de Kraker MEA, Jarlier V, Monen JCM, Heuer OE, van de Sande N, Grundmann H. The changing epidemiology of bacteraemias in Europe: trends from the European Antimicrobial Resistance Surveillance System. Clin Microbiol Infect. 2013;19(9):860-8.

3. Van Der Steen M, Leenstra T, Kluytmans JAJW, Van Der Bij AK. Trends in expanded-spectrum cephalosporin-resistant Escherichia coli and Klebsiella pneumoniae among Dutch clinical isolates, from 2008 to 2012. PLoS One. 2015;10(9):e0138088.

4. Vihta K-D, Stoesser N, Llewelyn MJ, Quan TP, Davies T, Fawcett NJ, et al. Trends over time in Escherichia coli bloodstream infections, urinary tract infections, and antibiotic susceptibilities in Oxfordshire, UK, 1998-2016: a study of electronic health records. Lancet Infect Dis. 2018;18(10):1138-49.

5. Werkgroep Infectiepreventie. WIP Richtlijn Meticilline-resistente Staphylococcus aureus (MRSA). 2012. https://www.rivm.nl/sites/default/files/2018-11/121205\%20MRSA\%20v1a\%20def.pdf. Accessed 20 Feb 2020.

6. Vos MC, Ott A, Verbrugh HA. Successful search-and-destroy policy for methicillin-resistant Staphylococcus aureus in The Netherlands. J Clin Microbiol. 2005;43(3):2034-5. 
7. Vos MC, Behrendt MD, Melles DC, Mollema FPN, de Groot W, Parlevliet G, et al. 5 years of experience implementing a methicillin-resistant Staphylococcus aureus search and destroy policy at the largest university medical center in the Netherlands. Infect Control Hosp Epidemiol. 2009;30(10):977-84.

8. van Rijen MML, Bosch T, Heck MEOC, Kluytmans JAJW. Meticillin-resistant Staphylococcus aureus epidemiology and transmission in a Dutch hospital. J Hosp Infect. 2009;72(4):299-306.

9. Vandenbroucke-Grauls CM. Methicillin-resistant Staphylococcus aureus control in hospitals: the Dutch experience. Infect Control Hosp Epidemiol. 1996;17(8):512-3.

10. Werkgroep Infectiepreventie. Beleid bij meticilline-resistente Staphylococcus aureus. Leiden; 1988.

11. Werkgroep Infectiepreventie. WIP Richtlijn Maatregelen tegen overdracht van bijzonder resistente micro-organismen (BRMO). 2005.

12. Werkgroep Infectiepreventie. WIP Richtlijn Bijzonder Resistente Micro-organismen (BRMO). 2013. https://www.rivm.nl/sites/default/files/2018-11/130424 BRMO.pdf. Accessed 20 Feb 2020.

13. von Elm E, Altman DG, Egger M, Pocock SJ, Gotzsche PC, Vandenbroucke JP. The Strengthening the Reporting of Observational Studies in Epidemiology (STROBE) statement: guidelines for reporting observational studies. PLoS Med. 2007;4(10):e296.

14. Tobi $H$, van den Berg PB, de Jong-van den Berg LTW. Small proportions: what to report for confidence intervals? Pharmacoepidemiol Drug Saf. 2005;14(4):239-47.

15. World Medical Association Declaration of Helsinki: ethical principles for medical research involving human subjects. JAMA. 2013;310(20):2191-4.

16. Kluytmans-van den Bergh MFQ, van Mens SP, Haverkate MR, Bootsma MCJ, Kluytmans JAJW, Bonten MJM, et al. Quantifying Hospital-Acquired Carriage of Extended-Spectrum Beta-LactamaseProducing Enterobacteriaceae Among Patients in Dutch Hospitals. Infect Control Hosp Epidemiol. 2018;39(1):32-9.

17. Bode LGM, Wertheim HFL, Kluytmans JAJW, Bogaers-Hofman D, Vandenbroucke-Grauls CMJE, Roosendaal R, et al. Sustained low prevalence of meticillin-resistant Staphylococcus aureus upon admission to hospital in The Netherlands. J Hosp Infect. 2011;79(3):198-201.

18. Weterings V, Veenemans J, van Rijen M, Kluytmans J. Prevalence of nasal carriage of methicillinresistant Staphylococcus aureus in patients at hospital admission in The Netherlands, 2010-2017: an observational study. Clin Microbiol Infect. 2019;25(11):1428.e1-1428.e5.

19. van den Bunt G, van Pelt W, Hidalgo L, Scharringa J, de Greeff SC, Schurch AC, et al. Prevalence, risk factors and genetic characterisation of extended-spectrum beta-lactamase and carbapenemaseproducing Enterobacteriaceae (ESBL-E and CPE): a community-based cross-sectional study, the Netherlands, 2014 to 2016. Eurosurveillance. 2019;24(41).

20. Dautzenberg MJ, Ossewaarde JM, de Kraker ME, van der Zee A, van Burgh S, de Greeff SC, et al. Successful control of a hospital-wide outbreak of OXA-48 producing Enterobacteriaceae in the Netherlands, 2009 to 2011. Eurosurveillance. 2014;19(9):20723.

21. Reuland EA, Overdevest ITMA, Al Naiemi N, Kalpoe JS, Rijnsburger MC, Raadsen SA, et al. High prevalence of ESBL-producing Enterobacteriaceae carriage in Dutch community patients with 
gastrointestinal complaints. Clin Microbiol Infect. 2013;19(6):542-9.

22. Zhou X, Garcia-Cobos S, Ruijs GJHM, Kampinga GA, Arends JP, Borst DM, et al. Epidemiology of Extended-Spectrum beta-lactamase-Producing E. coli and Vancomycin-Resistant Enterococci in the Northern Dutch-German Cross-Border Region. Front Microbiol. 2017;8:1914.

23. van den Braak N, Ott A, van Belkum A, Kluytmans JA, Koeleman JG, Spanjaard L, et al. Prevalence and determinants of fecal colonization with vancomycin-resistant Enterococcus in hospitalized patients in The Netherlands. Infect Control Hosp Epidemiol. 2000;21(8):520-4.

24. van den Bunt G, Top J, Hordijk J, de Greeff SC, Mughini-Gras L, Corander J, et al. Intestinal carriage of ampicillin- and vancomycin-resistant Enterococcus faecium in humans, dogs and cats in the Netherlands. J Antimicrob Chemother. 2018:607-14.

25. Otter JA, Mutters NT, Tacconelli E, Gikas A, Holmes AH. Controversies in guidelines for the control of multidrug-resistant Gram-negative bacteria in EU countries. Clin Microbiol Infect. 2015;21(12):105766.

26. Tacconelli E, Cataldo MA, Dancer SJ, De Angelis G, Falcone M, Frank U, et al. ESCMID guidelines for the management of the infection control measures to reduce transmission of multidrug-resistant Gram-negative bacteria in hospitalized patients. Clin Microbiol Infect. 2014;20 Suppl 1:1-55.

27. Gardam MA, Burrows LL, Kus J V, Brunton J, Low DE, Conly JM, et al. Is surveillance for multidrugresistant enterobacteriaceae an effective infection control strategy in the absence of an outbreak? J Infect Dis. 2002;186(12):1754-60.

28. Bootsma MCJ, Diekmann O, Bonten MJM. Controlling methicillin-resistant Staphylococcus aureus: quantifying the effects of interventions and rapid diagnostic testing. Proc Natl Acad Sci USA. 2006;103(14):5620-5.

29. Wertheim HFL, Vos MC, Boelens HAM, Voss A, Vandenbroucke-Grauls CMJE, Meester MHM, et al. Low prevalence of methicillin-resistant Staphylococcus aureus (MRSA) at hospital admission in the Netherlands: the value of search and destroy and restrictive antibiotic use. J Hosp Infect. 2004;56(4):321-5.

30. Souverein D, Houtman P, Euser SM, Herpers BL, Kluytmans J, Den Boer JW. Costs and Benefits Associated with the MRSA Search and Destroy Policy in a Hospital in the Region Kennemerland, The Netherlands. PLoS One. 2016;11(2):e0148175.

31. Clancy M, Graepler A, Wilson M, Douglas I, Johnson J, Price CS. Active screening in high-risk units is an effective and cost-avoidant method to reduce the rate of methicillin-resistant Staphylococcus aureus infection in the hospital. Infect Control Hosp Epidemiol. 2006;27(10):1009-17.

32. Lekkerkerk WSN, Sande-Bruinsma N van de, van der Sande MAB, Tjon-A-Tsien A, Groenheide A, Haenen $A$, et al. Emergence of MRSA of unknown origin in the Netherlands. Clin Microbiol Infect. 2012;18(7):656-61.

33. de Greeff SC, Mouton JW. Nethmap 2019. Consumption of antimicrobial agents and antimicrobial resistance among medically important bacteria in the Netherlands. 2019. 
34. Donker T, Bosch T, Ypma RJF, Haenen APJ, van Ballegooijen WM, Heck MEOC, et al. Monitoring the spread of meticillin-resistant Staphylococcus aureus in The Netherlands from a reference laboratory perspective. J Hosp Infect. 2016;93(4):366-74.

\section{Additional Files}

\section{Additional file 1:}

Table S1. Details of the MDRO risk assessment (DOC $15 \mathrm{~kb})$

\section{Additional file 2:}

Table S2. Microbiology culture classification (DOC 14 kb)

\section{Additional file 3:}

Table S3. Definitions multi-drug resistant organisms (MDRO) (DOC $15 \mathrm{~kb}$ )

\section{Additional File 4:}

Table S4. Trends over time (DOC $14 \mathrm{~kb})$

\section{Additional File 5:}

Table S5. Prevalence of new identified MDRO carriage per risk assessment question (DOC $15 \mathrm{~kb}$ )

\section{Additional File 6:}

Table S6. Types of multi-drug resistant organisms (MDRO) identified in clinical cultures during hospital stay, stratified for negative and positive risk assessment upon hospital admission and for hospitalacquired bloodstream infections (BSI) (DOC $23 \mathrm{~kb}$ )

Table S7. Types of multi-drug resistant organisms (MDRO) identified in hospital-acquired bloodstream infection (BSI) episodes caused by MDRO, stratified for negative and positive risk assessment upon hospital admission. (DOC $23 \mathrm{~kb}$ )

Table S8. Clinical culture types positive for a multi-drug resistant organism (MDRO) (DOC $23 \mathrm{~kb}$ )

\section{Figures}




\section{January 2015 - 1 Augustus 2019}

174,529 Admissions with one or more MDRO riskassessments on day of hospitalization

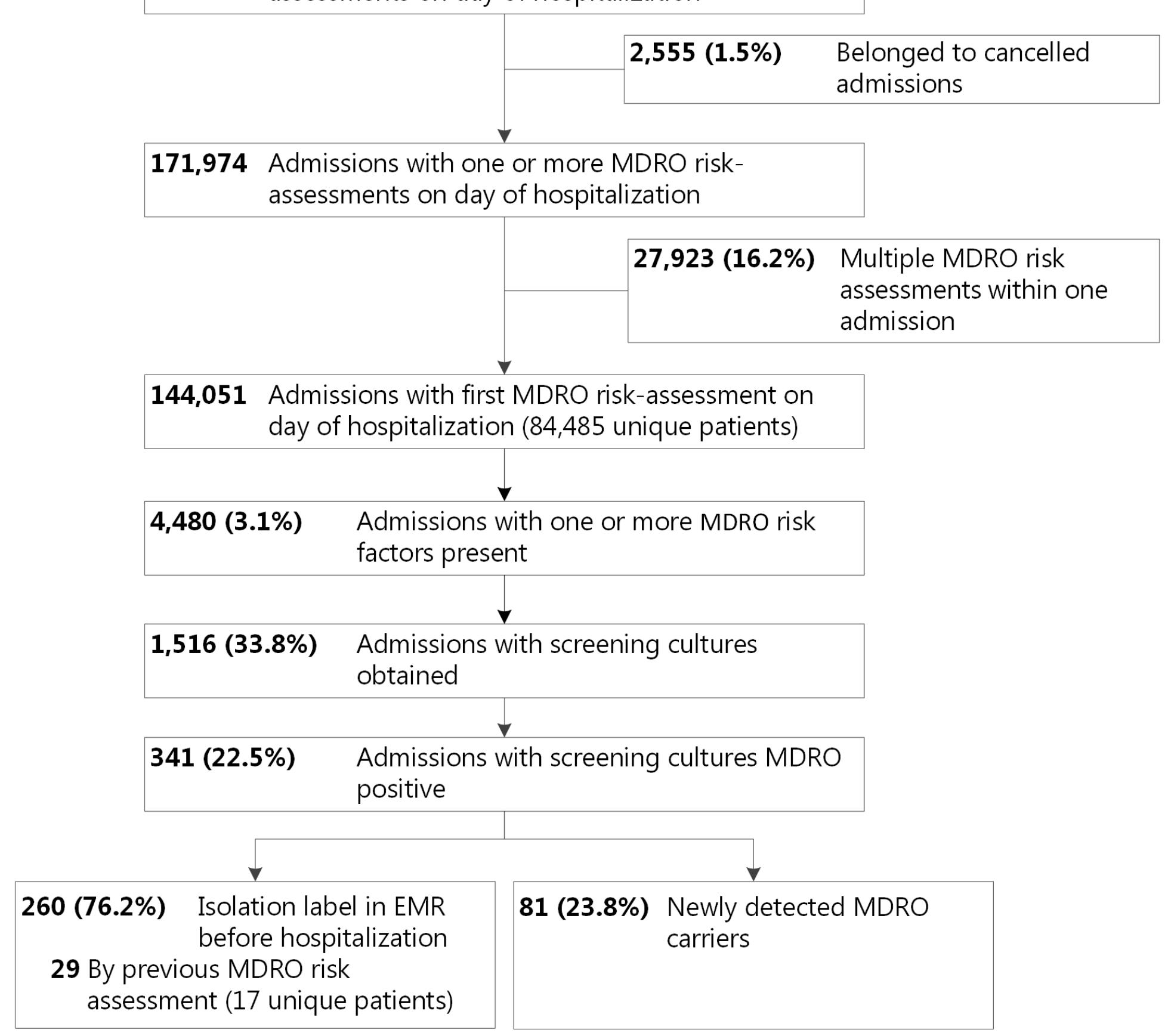

\section{Figure 1}

Study flowchart.

\section{Supplementary Files}

This is a list of supplementary files associated with this preprint. Click to download. 
- AdditionalFile2.docx

- AdditionalFile5.docx

- AdditionalFile4.docx

- AdditionalFile6.docx

- AdditionalFile1.docx

- AdditionalFile3.docx 